\title{
Simplifying the Automation of Manual Painting Processes
}

\section{A new motion tracking software} and hardware system makes programming painting robots easier. It allows manual painting processes to be automated with relatively little effort.

W riting programs for painting individual components using robots can be very time-consuming. ABB has developed an alternative solution that saves both time and money. The product goes under the name Simplified Robot Programming (SRP) and reduces the overall time needed for programming to just a few minutes. The programs can easily be amended at a later date on the PC. Using the latest motion tracking technology, SRP converts the skilled movements of the painter into a program. Instead of a real spray gun, the painter uses the Teach Handle, which resembles a traditional spray gun. A laser light shows the movements of the user and the virtual application of the paint. The software records the speed, accuracy and fluidity of the painter's move-

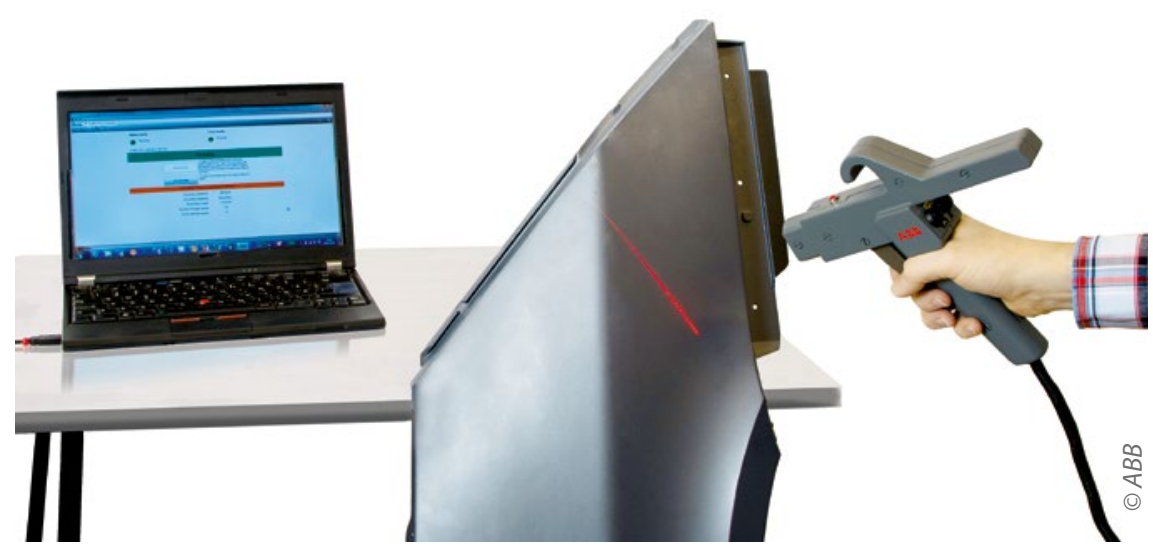

The motion tracking software and hardware package converts the skilled movements of the painter into a program. The data that is recorded can then be edited and validated on a PC.

ments and converts the results into code. Even someone with no programming experience can easily edit the code produced by the software on the PC. After this the robot can begin painting immediately, regardless of whether the workpieces are fixed in position or moving past on a conveyor.

\section{Programming tools replace}

paint guns

SRP is a software and hardware package that tracks coordinates and movements quickly. As already explained, the user can edit the processes that have been recorded in RobView, a PC program developed by $\mathrm{ABB}$ that is used to visualise, commission, control and monitor fully automated paint shops. It also offers a range of functions for operating one or more painting robots and the accompanying paint application equipment.

\section{Contact:}

ABB Automation GmbH, Friedberg, Germany,

Bettina Neubauer,

Tel. +49 603185 104, bettina.neubauer@de.abb.com

www.abb.de/robotics \section{Q ADAPTA}

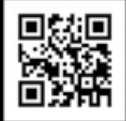

